\title{
Utilizing Multi-Hop Neighbor Information in Spectrum Allocation for Wireless Networks
}

\author{
Rongsheng Huang, Student Member, IEEE, and Yuguang Fang, Fellow, IEEE
}

\begin{abstract}
Spectrum sharing is a crucial issue to the overall throughput performance of multi-hop wireless networks. Traditional distributed random Medium Access Control (MAC), such as IEEE 802.11, lacks of efficiency of spectrum usage, while centralized scheduling is not practical for large scale ad hoc networks. It is observed that for multi-hop wireless networks, it is hard to resolve the scheduling conflict, and most distributed algorithms consider the neighbors' traffic independent of each other and ignore the multi-hop nature of flows, leading to the spectrum wastage and inefficiency. By incorporating the multihop nature of flows, we propose a new distributed scheme based on IEEE 802.11 standard, namely "2-hop MAC". Nodes collect traffic dependency information as well as traffic demand information from neighbors and allocate spectrum distributedly with the knowledge of more accurate traffic demand of the nodes in the neighborhood. Moreover, we have also addressed the problem of the asymmetric neighborhood, which was ignored in most previous work. Finally, we introduce a new metric, namely, allocation inefficiency ratio (AIR), to evaluate the performance of distributed algorithms in multi-hop wireless networks. Extensive simulation study shows that our proposed scheme can significantly improve the network performance and spectrum efficiency.
\end{abstract}

Index Terms-MAC, multi-hop, spectrum allocation, distributed resource allocation.

\section{INTRODUCTION}

$\mathbf{T}$ HE surprisingly poor performance of multi-hop wireless networks has attracted more and more attentions in the literature. During recent years, new transmission techniques are sprouting quickly. However, the traffic rate in multi-hop wireless networks is not increasing accordingly. Usually, when the scale of the networks becomes large, due to the increasing interference and the increasing number of intermediate hops of flows, the end-to-end throughput performance starts to deteriorate. Gupta and Kumar theoretically characterize this in [1]. However, for practical multi-hop wireless networks, such as WMNs, WSNs and some battle-field ad hoc networks, there is plenty of room to improve the throughput performance since the current poor throughput performance of these networks is mainly due to the inefficiency of spectrum usage. Most of

Manuscript received February 27, 2009; revised April 17, 2009; accepted May 11, 2009. The associate editor coordinating the review of this paper and approving it for publication was Y.-B. Lin.

The authors are with the Department of Electrical and Computer Engineering, University of Florida, Gainesville, FL 32611, USA. Y. Fang is also a Chanjiang Scholar Chair Professor with the National Key Laboratory of Integrated Services Networks, Xidian University, Xi'an 710071, China (email: \{rshuang@, fang@ece.\}ufl.edu).

This work was partially supported by the National Science Foundation under grant CNS-0721744. The work of Fang was also partially supported by the 111 Project under Grant B08038.

Digital Object Identifier 10.1109/TWC.2009.090246 recent research works are focusing on the spectrum efficiency for this reason.

As the de facto standard of most of the multi-hop wireless networks, IEEE 802.11 was originally designed for the singlehop Wireless LANs. Its performance in multi-hop scenarios is much below our expectation due to its blindly-contending and mechanically forwarding properties [2] [3]. This random access property of IEEE802.11 is one of the major reasons for the inefficient resource usage. Scheduling-based protocols, like IEEE 802.16, can provide better spectrum efficiency because it does not require nodes to contend for the channel before each transmission with the assumption of relatively constant traffic flows. However, since this type of protocols needs a fixed frame format which is vulnerable to the scheduling conflict, it is not suitable for the multi-hop wireless networks.

Random access MAC provides a roughly fair mechanism for wireless nodes to access the medium. The effort of differentiating the uplink and downlink resource allocation has been first applied to WLANs in [4] because of the observation that as the central point, APs should occupy more resource than other nodes. To achieve better performance in multi-hop networks, several previous schemes attempt to break the fairness by prioritization, [5], [6], [7]. These schemes heuristically search for better spectrum sharing mechanism among wireless nodes, by differentiating the forwarding priority according to the priority tags of packets or flows. However, when the traffic pattern is more complicated, these schemes cannot guarantee significant performance improvement.

On the other side, with centralized approaches, schedulingbased MAC can allocate the resource in a more efficient way. This approach can find the optimal solution with knowledge of the topology and traffic when the network is not large. However, for large-scale networks, this approach becomes infeasible due to the NP-hardness and the difficulty of information collection. Therefore, the distributed scheduling approaches are proposed to address this dilemma, [8], [9]. These two approaches give us the insight of how good performance the networks can achieve. However, they always require a perfect scheduling, a MAC with no collision and no hidden/exposed terminals, which is almost impossible in multi-hop wireless networks. Previous distributed scheduling schemes also ignore the multi-hop nature of flows in multi-hop networks, which causes a lot of wastage in spectrum allocation. Moreover, within a neighborhood range, different neighbors can sense different condition of channels, resulting in potential conflicts of distributed scheduling, which is difficult to solve in distributed scheduling schemes.

In this paper, we propose a new MAC with a different 
spectrum allocation mechanism based on IEEE802.11. Similar to previous distributed approaches, the efficient spectrum usage of this scheme comes from the collection of neighbors' traffic information. Different from previous works, our scheme collects traffic dependency information from neighbors as well. By addressing "asymmetric neighborhood" and "traffic dependency" issues, this paper gives a comprehensive way to improve the throughput performance in multi-hop wireless networks. Upon the observation of efficiency of spectrum allocation, a new metric called "Allocation Inefficiency Ratio (AIR)" is introduced for better evaluating the proposed scheme.

The rest of this paper is organized as follows. Section II introduces the related works. Section III discusses the spectrum usage issues in multi-hop wireless networks. Section IV describes the proposed schemes. Section V provides the evaluation study for our scheme. Finally, conclusion is drawn in Section VI.

\section{RELATED WORKS}

In the literature, there are two types of research aiming at improving the performance of multi-hop wireless networks ditributedly. One is the heuristic approach based on the existing random access MAC, such as IEEE802.11. The other assumes a perfect MAC and gives out distributed scheduling algorithm. The former approach proposes practical ways to differentiate the medium access probability, thus a better spectrum usage can be achieved. The latter one takes the traffic load of each neighbor as input and tries to optimize the overall throughput performance in a distributed manner. We start the survey with the first approach.

In [5], forwarded packets are given higher priority than upstream packets by a shorter IFS, thus the packet accumulation at the forwarding nodes is alleviated and the delivery failure due to forwarding congestion can be reduced. Yang and Vaidya tried to ensure medium access for high priority source stations in their priority scheduling scheme [6]. Two narrow band busy-tone signals are used to ensure medium access for high priority nodes. Kanodia et al. further proposed a distributed priority scheme to differentiate the different packets' priority in transmission [7]. Accordingly, the forwarded packets have their priority increased for the same reason as addressed in [5]. All the above works attempt to improve the performance by introducing differentiation among different nodes, different packets, or different flows. However, such differentiations cannot improve the overall performance of the system remarkably when the traffic and topology become more complicated or when flows cannot be prioritized. IFA scheme proposes another way of spectrum usage allocation [10] based on the random access MAC. The neighbors' traffic information are collected and the spectrum resource is allocated accordingly. By throttling input traffic to its system wide fair time share, random access MAC can greatly improve the fairness and throughput performance. However, the traffic demand of each node simply uses the value of arrived traffic and only parking lot topologies are analyzed.

The distributed scheduling approach focuses on the overall throughput performance analytically since they are derived from centralized scheduling algorithms. The scheme in [11] requires each node to collect each neighbor's queue-length information and use a probability $\alpha$ to transmit its packets. The probability $\alpha$ is derived from the relationship of each node's queue-length with its neighbors'. This paper proves that when this policy of distributed scheduling is applied, the largest capacity region $\Omega$ can be achieved with an efficiency ratio $\gamma$. Lin's work has taken multi-channel and routing into consideration besides each neighbors' queue-length [8]. According to the calculated contention cost, radio cost and congestion level, which are derived from neighbors' queuelength, channel condition and nodes' other information, each node decides the assignment of packets to different channels and different slots. This paper also shows that the provided distributed algorithm is provably efficient, which means that a provable fraction of the maximum system capacity can be achieved.

Although these works provide theoretical results of overall throughput performance, the assumptions of a perfect MAC and the lack of a real protocol support prevent them from transiting the theoretical results to a practical protocol. Moreover, when all queues are full and the traffic is backlogged due to over-injection of traffic, these distributed scheduling algorithms cannot address the congestion problem.

Furthermore, the analysis of these works do not consider the multi-hop nature of flows. They consider the traffic load (queue length) independently while the inherent traffic correlation among neighboring nodes is ignored. Similar to [10], they take the arrived traffic as the traffic demand. We will discuss this ignored issue in the later section of "traffic dependency".

No matter which approach is concerned, the purpose is to efficiently utilize the spectrum resource. The reason of the collection of neighbors' information is to let each node efficiently share the spectrum resource. For multi-hop wireless networks, only neighbors' traffic load information is not enough for the ideal spectrum usage allocation.

\section{Spectrum Usage IsSUeS IN MUlti-Hop Wireless NETWORKS}

\section{A. Ideal Spectrum Usage}

Assuming a random access MAC, we hereby give an example of the spectrum inefficiency caused by fair access to the medium in multi-hop wireless networks. Fig. 1 shows 3 flows in a simple 4-node 2-hop topology. Flow 1 and 2 share the same forwarding node, Node 3. With IEEE802.11 protocols, Node 3 can share $1 / 3$ of the channel capacity like Node 1 and Node 2, with Node 4 only receiving. In this case, a part of the channel capacity Node 1 and Node 2 hold is wasted because of Node 3's delivery limit. Consequently, we can expect that when the traffic consists of stable and continuous flows, adjusting each node' medium access can bring significant improvement of the throughput performance.

Usually the performance of multi-hop wireless networks can be evaluated by two metrics: fairness and end-to-end throughput. Suppose the flow demand vector is $\left\{F_{1}, F_{2}, F_{3}, \ldots, F_{N}\right\}$, where $\mathrm{N}$ denotes the number of existing flows. With a certain scheme, each flow can achieve a flow rate according to the flow allocation vector $\left\{f_{1}, f_{2}, f_{3}, \ldots, f_{N}\right\}$. A perfectly fair 


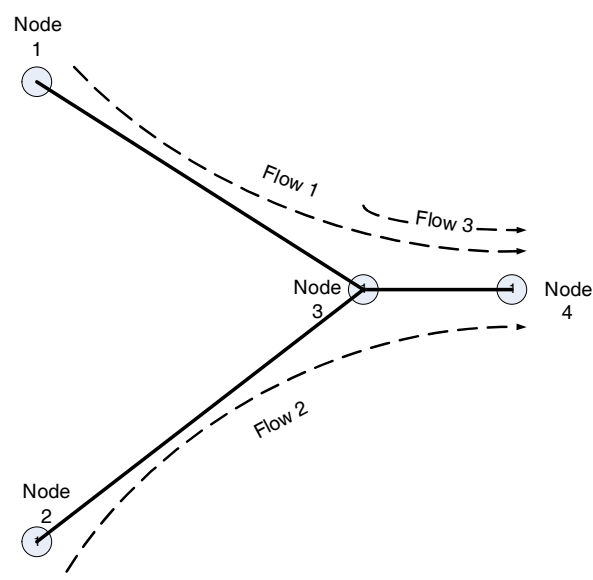

Fig. 1. Inefficiency of Fair Random Access

allocation means the proportion of each allocated flow rate to its demand is identical, as discussed in [10]. However, this does not guarantee an optimum allocation since the aggregate end-to-end throughput is not considered. Using max-min delivery ratio as the criteria as in [12] includes the end-to-end throughput into consideration. In this paper, the delivery ratio is defined as follows.

$$
\zeta_{i}=\frac{f_{i}}{F_{i}}
$$

The objective is:

$$
\max \left\{\min _{i \in \mathbb{F}}\left\{\zeta_{i}\right\}\right\}
$$

Let us consider Fig. 1 again. When legacy IEEE802.11 is applied, each active node obtains the same share of wireless channel. The overall end-to-end throughput of this small network is roughly $1 / 3$, with each flow taking $1 / 9$ equally. When scheduling is used and no fairness is concerned, the maximum overall end-to-end throughput can reach 1 when Flow 3 takes all the spectrum resource. However, the minimum flow rate is 0 , with flow 1 and 2 totally starved. If we apply the max-min criterion to this simple topology, we can obtain the ideal spectrum usage allocation simply by observation. The maximum overall end-to-end throughput is $3 / 5$, with each flow taking $1 / 5$ and the remaining $2 / 5$ taken by the first hop of Flow 1 and Flow 2. Each flow obtains the same flow rate 1/5, thus the max-min goal is achieved.

For complicated topologies, it is not easy to find the ideal allocation by simple observation since more complicated interference and frequency reuse need to be considered. When the knowledge of the whole topology and flow information is given, there are many algorithms to find this ideal allocation while NP-hardness is a big obstacle for the solutions. The distributed scheduling proposes to utilize the queue-length or traffic load information of neighboring nodes as the input of distributed scheduling algorithm [8], [9], [11]. The distributed scheduling is in nature another type of spectrum allocation. The intuition is straightforward in that each node can get its spectrum share based on the proportion of its traffic load to the total traffic load within the interference range.

However, most of the distributed algorithms have a hidden assumption that each node's traffic load is independent, which does not hold when multi-hop flows exist.

\section{B. Traffic Dependency}

When distributed algorithms are applied in multi-hop ad hoc networks, as in previous papers [8], [9], [10], [11], the traffic demand of each node is assumed to be the local traffic input. However, this traffic input depends on not only the arrival traffic from local upper layer entities, but also the forwarding requirement from the upstream nodes, which depends on current scheduling or spectrum allocation. Therefore, using the local traffic as the input of the algorithm can make the spectrum allocation deviated from the ideal one. Previous papers ignore this traffic relationship in their algorithms, which we term it as "traffic dependency".

We use Fig. 1 as the example to illustrate the problem of ignoring traffic dependency. Suppose Node 1, 2 and 3 have the same original traffic load, valued as 1 , and Node 3 needs to forward the traffic from both Node 1 and 2. Suppose each node collects only the arrival traffic information of each neighbor and allocates the spectrum accordingly. The initial arrival traffic rate of each node is assumed to be the result of random access. The result turns out to be far deviated from the ideal result, with each of Node 1 and 2 occupying $3 / 11$ of the channel capacity and Node 3 occupies $5 / 11$. We can see that Node 3's allocated resource cannot even cover its forwarding requirement, and thus a big wastage is created. This allocation deviation comes from the ignorance of traffic dependency of neighboring nodes.

Traffic dependency comes from flows' multi-hop delivery property. Li has raised similar concerns of this "inherent correlation" of upstream and downstream sub-flows in [13]. For centralized algorithms, the central point is assumed to have all the knowledge of existing flows. Therefore, all flow and topology information, thus the traffic dependency information, can be acquired and processed at the central point. For distributed algorithms, the information mentioned above is difficult to obtain for individual nodes. Fortunately, traffic dependency information can be obtained by the information exchange of forwarding request from upstream nodes (nodebased information), which bypasses the flow-based approach.

\section{Allocation Inefficiency Ratio}

In multi-hop networks, end-to-end throughput can only indicate the absolute value of deliverable traffic given the traffic pattern and topology. It cannot indicate the performance of applied schemes because this value depends on the traffic pattern and topology heavily. We need a metric which can characterize not only a system's absolute performance but also the efficiency of the scheme applied. To better evaluate how the spectrum usage allocation is, we propose a new metric, called allocation inefficiency ratio (AIR). It is defined as the ratio between the air-time of the dropped traffic amount due to inefficient spectrum allocation and the total air-time allocated

$$
A I R=\frac{\sum_{i \in \mathbb{N}} \frac{T_{i}}{P H Y_{i}}-\sum_{j \in \mathbb{F}} \sum_{k \in \mathbb{K}_{j}} \frac{T e 2 e_{j}}{P H Y_{j, k}}}{\sum_{i \in \mathbb{N}} \frac{T_{i}}{P H Y_{i}}},
$$

where $\mathbb{N}$ is the node set, $T_{i}$ is the outflow rate of node $i, \mathbb{F}$ is the flow set, $\mathbb{K}_{j}$ is the hop set of flow $j, T e 2 e_{j}$ is the end-toend rate of flow $j$, and $P H Y_{j, k}$ is the physical layer rate of flow $j$ at hop $k$. 
Apparently, this metric gives us the knowledge of how much allocated spectrum is wasted. This wastage mainly comes from allocation discrepancy at different hops for one flow. A good spectrum allocation scheme should have an AIR with the value of 0 . We can verify this claim according to the AIR calculation in above examples. The AIR value in the schemes in [9], [11], [8], [10], which ignore traffic dependency, is $8 / 33$. This means $8 / 33$ of the allocated spectrum is wasted because the corresponding traffic amount originally transmitted is not finally delivered.

\section{Asymmetric Neighborhood}

Due to multi-hop topology, each node has overlapping and non-identical neighborhood which may make information asymmetric. This information asymmetry is sometimes undesirable to a distributed scheme. For example, some node senses a relatively idle channel and decides to use its demanded traffic rate. However, some of its neighbors sense the channel in busy status instead. Therefore, conflicts are introduced when distributed scheduling or distributed allocation is implemented. Previous works have not provided solutions for this problem. Incorporating 2-hop neighbors' traffic information will not help to solve this problem because the sensed total traffic load is not exchanged and can be different from node to node.

\section{Proposed Scheme}

\section{A. Overview of The Proposed Scheme}

For practical ad hoc networks, like WMNs or WSNs or other military ad hoc networks, the traffic is not totally ad hoc. Usually traffic aggregates at some points or areas with certain patterns. In this paper, we assume that the multi-hop wireless networks which we concern have certain traffic patterns and the flows inside have relatively stable traffic load. For these ad hoc networks, we design an efficient MAC which can utilize the limited spectrum resource in a more efficient way.

The basic procedure can be described as follows. Each node is required to broadcast its traffic demand to its neighbors, which is the same as previous works. Meanwhile, each node is required to notify its neighbors about the traffic dependency between them, which differentiates our work from others. Afterwards, each node allocates the spectrum individually according to the information collected and apply the calculated traffic rate to its transmission.

Traffic dependency information from different neighbors affects the estimation of accurate traffic load in different ways. Three different roles of neighbors are defined in this paper. When one neighbor has traffic for the current node to forward, we name this role as upstream neighbor. Similarly, when the current node has traffic for its neighbors to forward, these neighbors are called downstream neighbors. Other neighbors are called uncorrelated neighbors. It should be noted that only when traffic demand of neighbors are correlated with the current node, are these neighbors to be seen as upstream neighbors or downstream neighbors. Therefore, one node sees the neighbors who have traffic ending at itself as uncorrelated neighbors because the traffic ending at itself will not affect its traffic demand.
The traffic demand from each node consists of two parts: the traffic that requires to be forwarded from its upstream neighbors and the traffic originated from its upper layer locally. It can be expressed by the following formula:

$$
T D_{i}=T D O_{i}+\sum_{j \in \mathbb{N}_{i}} T D f w \text { ding }_{j, i}
$$

where $T D_{i}$ is the traffic demand, $T D O_{i}$ is the traffic originated from local upper layer, $T D f w_{d i n g} g_{j, i}$ is the traffic that requires to be forwarded from its upstream neighbor $j$ and $\mathbb{N}_{i}$ is the set of neighbors for node $i$. The latter part is dependent with its neighbors' traffic demand and the former part is independent. Therefore, an accurate traffic demand of one node should be based on the knowledge of all upstream neighbors' traffic dependency information. In this scheme, upstream nodes should notify their downstream neighbors about their forwarding request. Consequently, the downstream nodes update their traffic demand accordingly. The knowledge of accurate local traffic demand is not enough for ideal spectrum allocation. It is also important to acquire the correct traffic demand of the neighbors, $T D_{i}$. When downstream nodes broadcast their new traffic demands, since the downstream neighbors' traffic includes forwarding requirement from the upstream nodes, the upstream nodes should be able to extract the dependent traffic from the messages, thus the pure change of the original traffic of the downstream nodes can be known. This knowledge is important in obtaining the accurate traffic demand of neighbors. Since each node considers both onehop upstream neighbors' and one-hop downstream neighbors' traffic dependency information, we name this scheme "2-hop MAC".

It is obvious that due to complicated traffic patterns, each neighbor can play different roles simultaneously. However, we keep these terms in this section to illustrate the scheme clearly.

In this scheme, one node's traffic change will affect the traffic demand of correlated neighbors. The change of one node's traffic demand is passed to other nodes as if there is no channel limit. In this way, the traffic demand in this scheme can reflect the true traffic demand of the neighborhood. Therefore, the spectrum allocated to one packet in the current hop will also be allocated to this packet in other hops, and thus the bandwidth waste due to allocation discrepancy is reduced from the beginning of the allocation phase. In the spectrum allocation phase, the channel limit comes into play to give each node the identical allocation ratio, and thus the fairness is well-addressed. There exists another type of traffic dependency when CSMA/CA is applied, because the receiving nodes need to send CTS or ACK messages upon receiving packets from the sending nodes. In our spectrum allocation, we count this part into sending nodes' spectrum usage.

If each node in the neighborhood has the same information as each other, the distributed spectrum allocations should be identical. In multi-hop scenarios, it is common that neighbors have different neighborhood, thus the distributed spectrum allocations are probably different. If the total spectrum allocation does not exceed the channel limit, the distributed algorithm can be acceptable even though some fairness is sacrificed. However, spectrum allocation conflicts usually exist with the asymmetric neighborhood. The proposed scheme uses 
a feedback mechanism to regulate the neighboring nodes' traffic rate from over-injection.

When a single rate is used, fairness requires the real traffic rate of each node is proportional to the ratio between its traffic demand and the total traffic demand. For multi-rate support, the fairness is based on air-time as previous papers, such as [14]. Each node's traffic load is expressed by the traffic rate. Based on the links' achievable PHY rate, this traffic load can be converted to air time, which is used for fair spectrum allocation in local area. We ignore how the rate adaptation mechanism is implemented in this paper. We also simply assume that during one period each node uses one constant PHY rate in one link to its neighbors. The proposed scheme requires the air-time fairness among neighbors, which means the air-time fraction of each node's allocated traffic rate is proportional to the ratio between its air-time of traffic demand and the summation of the air-time of each node's traffic demand. This can be expressed as formula 5 .

$$
\frac{\frac{R_{i}}{P H Y_{i}}}{\text { ChannelLimit }}=\frac{\frac{T D_{i}}{P H Y_{i}}}{\sum_{j \in \mathbb{N}} \frac{T D_{j}}{P H Y_{j}}}
$$

$R_{i}$ and $P H Y_{i}$ denote the determined traffic rate and physical layer rate for node $i$, respectively. We assume the channel limit in multi-rate environment to be a constant in this paper.

\section{B. Scheme Description}

In a real network, one node can play different roles of neighbors, downstream, upstream or uncorrelated neighbors, for its neighbors, due to the co-existence of different flows. Therefore, the proposed scheme assumes each neighbor as all possible types of neighbors. The messages from each neighbor are processed with a uniform procedure.

In this scheme, each node maintains three tables which record its own traffic information, its neighbors' traffic information and the traffic dependency information. Each node periodically gets knowledge of its original traffic load and forwarding traffic load from its upper layers and updates these tables. It also updates these tables when it receives/overhears messages from its neighbors. Each node calculates the achievable traffic rate for itself and its neighbors distributedly, with the algorithm which will be presented later. Regulation indicators are used to eliminate the over-injection of traffic due to allocation conflicts.

We denote the maximum normalized IEEE802.11 one-hop MAC throughput as $\eta_{C}$, which also means the maximum busy air-time fraction $\left(\eta_{C}=\frac{R_{C}}{P H Y}\right)$. The overhead incurred by the rate adaptation is assumed to be negligible. Related works, such as [15], can provide ways on how to determine the value of $\eta_{C}$.

In the following subsections, we first present the supporting data structure and messages' format. We then describe the detailed procedure and algorithm.

1) Data Structure and Message Format: Each node should maintain a parameter set as described in Table I which includes its own traffic information:

Each node should also maintain a table which records its neighbors corresponding information as the potential input of
TABLE I

LOCAL PARAMETER SET

\begin{tabular}{|l|l|}
\hline$T D O$ & $\begin{array}{l}\text { The traffic demand originated from upper layer lo- } \\
\text { cally }\end{array}$ \\
\hline$R$ & The node's allocated and broadcasted traffic rate \\
\hline$R^{*}$ & The node's adjusted traffic rate after regulation \\
\hline$T D$ & The node's traffic demand \\
\hline Status & $\begin{array}{l}\text { The status parameter used to indicate different mes- } \\
\text { sage requests }\end{array}$ \\
\hline
\end{tabular}

TABLE II

NeIGHBor PARAMETER SET

\begin{tabular}{|l|l|}
\hline$I D$ & Neighbor Address, as the ID of each record. \\
\hline Status & Neighbor Status: Active or inactive \\
\hline$R_{i}$ & Neighbor i's traffic rate \\
\hline$R_{i}^{*}$ & Neighbor i's regulated traffic rate \\
\hline$P H Y_{i}$ & Neighbor i's physic layer rate \\
\hline$T D t f w_{i}$ & $\begin{array}{l}\text { Forwarding requirement to neighbor i, the latest } \\
\text { value }\end{array}$ \\
\hline
\end{tabular}

distributed spectrum allocation. The detail information is listed in Table II.

The traffic dependency information is stored in an $(n+$ $1) \times(n+1)$ matrix $D$, with the local traffic demand included. $D_{i, j}$ means the forwarding requirement from node $i$ to node $j$. When $i$ equals to $j, D_{i, j}$ stores the original traffic excluding its forwarding demand from its neighbors in this neighbor set. Note that this original traffic demand may not purely be original. In node $i$ 's storage, if neighbor $j$ has some forwarding request from its neighbor $\mathrm{k}$, which is not a neighbor of node $i$, neighbor $j$ 's original traffic demand in the matrix includes this part of forwarding request. Obviously, we have the relationship as follows:

$$
T D_{i}^{\prime}=\sum_{j \in \mathbb{N}} D_{i, j}
$$

$T D_{i}^{\prime}$ in Formula 6 means the broadcasted version of $T D_{i}$. Throughout this paper, superscript ' stands for the broadcasted version and superscript ${ }^{*}$ stands for the regulated version. Apparently, matrix $D$ 's storage has some overlapped information with the former two tables. The difference is that matrix $D$ only stores the broadcasted version and the other two tables gather the latest information.

The broadcast messages should contain the following information: the traffic demand of the node $(T D)$, the traffic load/rate $(R)$ and the adjusted traffic $\operatorname{rate}\left(R^{*}\right)$ of the node, a list of traffic that the host needs the neighbor to forward $\left(T D t f w_{i}\right)$, an indicator that a traffic regulation is necessary or this regulation is to be deactivated. There is one more parameter, unit demand allocation $\varepsilon$, is implied in the broadcast messages.

$$
\varepsilon=\frac{R}{T D}
$$

This value is used to eliminate the conflicts due to asymmetric neighborhood, whose usage will be illustrated in a later section.

2) Procedure: The tables are updated according to local cross-layer notification or neighbors notification as mentioned above. The accurate traffic load of each node in the neighborhood is calculated according to these tables. Spectrum allocation is executed with the knowledge of traffic load and 
channel limit. Meanwhile, a check for regulation indicator or deactivation indicator is necessary to avoid the allocation conflicts due to asymmetric neighborhood.

For upstream neighbors, traffic demand is as broadcasted since no other nodes in the neighborhood can affect this value. However, local node's traffic demand is affected by its upstream neighbors. The local traffic demand calculation can be found in equation (4). For downstream neighbors, the traffic demand depends on local traffic change. It can be calculated as follows:

$$
T D_{i}^{\text {down }}=\sum_{j \in \mathbb{N}} D_{j, i}-T D t f w_{i}^{\prime}+T D t f w_{i}
$$

where $T D t f w_{i}^{\prime}$ can be found in matrix $D$ according to the current node index (If it is calculated by Node $k, T D t f w_{i}^{\prime}=$ $\left.D_{k, i}\right)$. Since each node can play different roles simultaneously, the overall traffic demand can be calculated in this way:

$$
T D_{i}=T D O_{i}+\sum_{j \in \mathbb{N}, j \neq i} D_{j, i}-T D t f w_{i}^{\prime}+T D t f w_{i}
$$

If local node index is not $i, T D O_{i}$ uses the value of $D_{i, i}$. We can see that $T D t f w_{i}$ changes when upstream neighbors' traffic changes, which embodies the traffic dependency. Through this way, the accurate traffic demand can be calculated by each node locally and the ideal spectrum allocation can be fulfilled.

The asymmetric neighborhood information can cause different spectrum allocation at different neighbors, leading to spectrum usage conflicts. When one node senses the total spectrum allocation by individual neighbors exceeding the channel limit, a regulation indicator is sent out. The regulation mechanism in this scheme uses the parameter of $\varepsilon$. This parameter stands for the uniform allocatable traffic air-time per unit traffic demand in air-time form. Each individual node allocates the traffic rate based on the air-time fairness, which means $\frac{\frac{R_{i}}{P H Y_{i}}}{\frac{T D i_{i}}{P H Y_{i}}}$ is identical for all nodes within its neighborhood from its point of view. When it senses that the conflicts happen and the total channel limit has been exceeded, if each of its neighbors can follow its $\varepsilon$ by applying $\varepsilon * T D_{i}$ to their traffic rate, the over-injection can be regulated. When the node senses the total traffic demand decreasing and the regulation is no longer necessary, a regulation deactivation indicator is required to recover the neighbors' traffic rate. Fig. 2 shows the flow chart.

Before every data transmission, if parameters in the record have been updated, a distributed spectrum allocation algorithm is carried out. When the node starts to transmit, it piggybacks its broadcast information in RTS or DATA packets. After broadcasting, the newly calculated values update the old record. If $T D, R$ and $T D t f w_{i}$ are not changed since last broadcasting, no information is attached to the data. The algorithm executed before broadcasting is shown in Fig. 3. The gross spectrum allocation can be briefly described as follows. If the total traffic does not exceed the channel traffic limit, $\eta_{C}$ etc., the node uses its required traffic rate. Otherwise, the node uses the traffic rate in proportion to the channel traffic limit. The basic allocation algorithm can be expressed in the following equation.

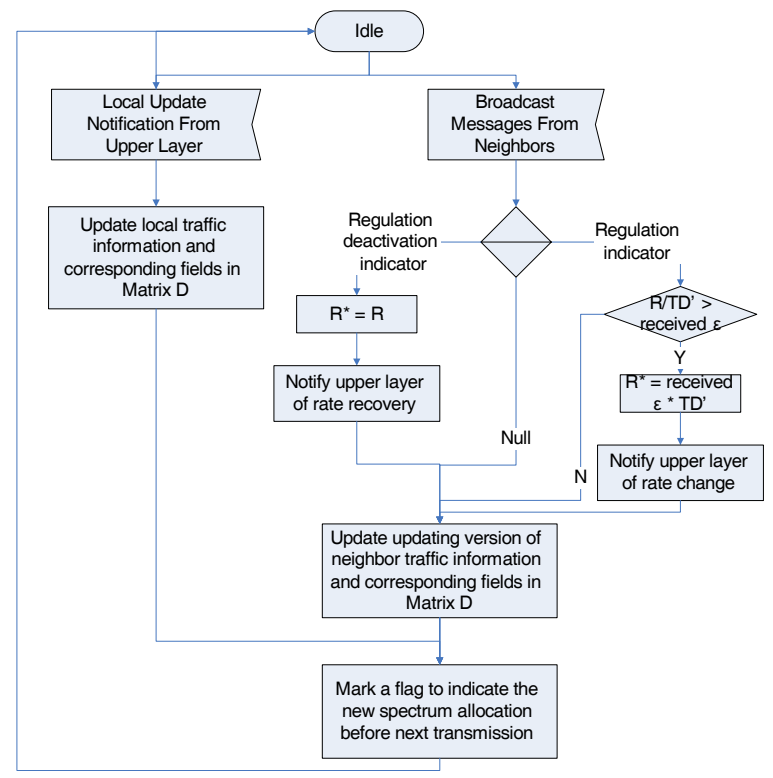

Fig. 2. Flow Chart for Message Processing

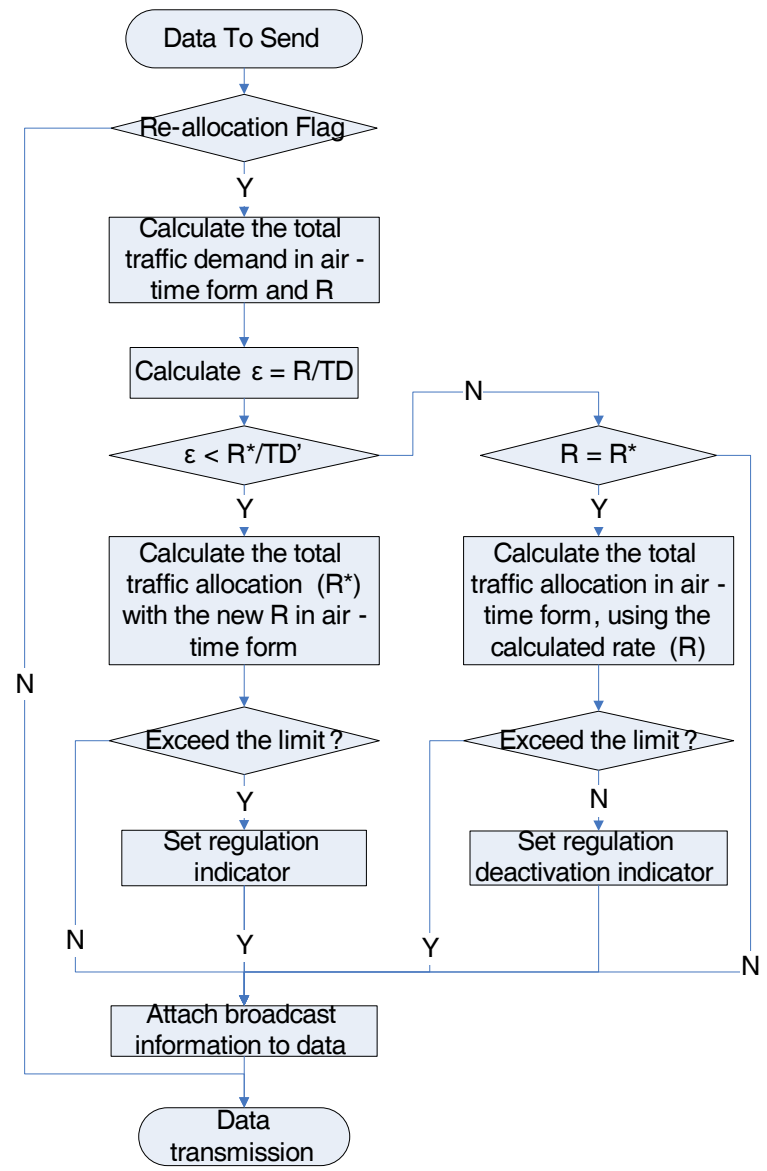

Fig. 3. Flow Chart for Spectrum Allocation

$R_{i}=\left\{\begin{array}{c}T D_{i}, \quad \eta_{C} \geq \frac{T D_{i}}{P H Y_{i}}+\sum_{j \in \mathbb{N}} \frac{D_{i, j}-T D t f w_{j}^{\prime}+T D t f w_{j}}{P H Y_{j}} \\ \frac{T D_{i}}{P H Y_{i}}+\sum_{j \in \mathbb{N}} \frac{D_{i, j}-T D t f w_{j}^{\prime}+T D t f w_{j}}{P H Y_{j}} \cdot \eta_{C}, \\ \eta_{C}<\frac{T D_{i}}{P H Y_{i}}+\sum_{j \in \mathbb{N}} \frac{D_{i, j}-T D t f w_{j}^{\prime}+T D t f w_{j}}{P H Y_{j}}\end{array}\right.$

If no information has been changed since last broadcasting, 
the spectrum allocation is not needed. If the total traffic demand does not exceed the channel limit, the spectrum allocation is not needed, either, though the traffic information change should be broadcasted.

It is worthwhile to note that although this scheme requires the modification of legacy IEEE802.11 messages' format and the regulation outgoing traffic, the algorithm is not complicated and it is feasible to upgrade the current 802.11 protocol to our scheme.

\section{Performance Evaluation}

There are two common metrics to judge the performance of a multi-hop MAC scheme, the end-to-end throughput and the fairness. In the proposed scheme, the spectrum sharing follows the proportions of each nodes' traffic demand. Although this fairness is based on nodes' traffic, the flows' fairness can be achieved when each node allocates its spectrum share based upon the flows' traffic proportions. We will evaluate the flows' fairness index in the following simulation. The end-to-end throughput is an important performance metric of multi-hop networks. However, it is not a good metric to evaluate the design of a scheme for the reason described above. Additionally, we adopt the AIR evaluation in this study.

As we mentioned above, the end-to-end throughput performance depends on the topology and the traffic pattern heavily. The end-to-end throughput varies when the topology or the traffic pattern is slightly changed. In this simulation, we construct 4 different and typical 3-hop (4-hop in the last topology) topologies for evaluation, as shown in Fig. 4. Since the benefits of the proposed scheme is more prominent when the traffic pattern is less homogeneous, we abandon the commonly-used grid topology. The circle nodes have original traffic while the rectangular nodes only forward traffic for the neighbors. The elliptical nodes only receive traffic. Consequently, there are both long-hop (3-hop or 4-hop) flows and short-hop (2-hop) flows in the constructed networks. In the last topology (topology (d)), there is a case for bi-directional flows. The original traffic demand is marked under each node. To illustrate the spectrum allocation result clearly, the traffic demand is set to exceed the channel limit, which is assumed to be $11 \mathrm{kbps}$.

For comparison purpose, we apply two other schemes to these topologies. One is random fair access MAC (RFA) and the other is a distributed scheduling scheme (DSS). RFA lets each node access the medium randomly, thus fairly (assumed). DSS requires each node collect the neighbors' traffic demand, however, with no consideration of traffic dependency and allocate spectrum accordingly. The traffic demand in this scheme is simply taken as the traffic arrival rate. Since the traffic arrival rate depends on a predefined scheduling output, we set the initial scheduling as fair medium access. Although the first two schemes lack of mechanisms to overcome the asymmetric neighborhood problem, in this simulation, we assume there exists a regulation mechanism which can regulate the traffic within each neighborhood so that it will not exceed the channel limit. The first two schemes require each node to deliver the traffic from different sources according to the arrival proportions. The overhead of all 3 schemes is ignored.

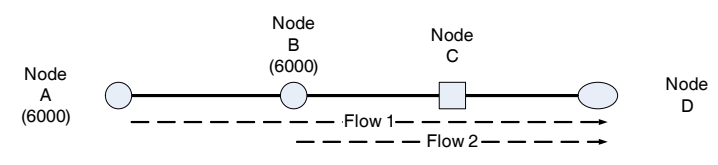

(a)

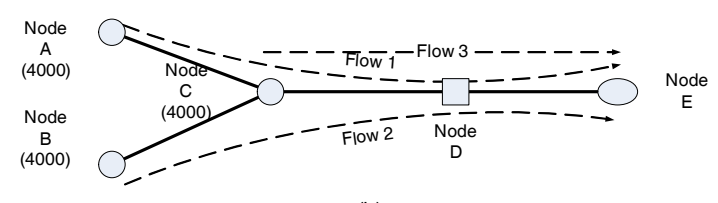

(b)

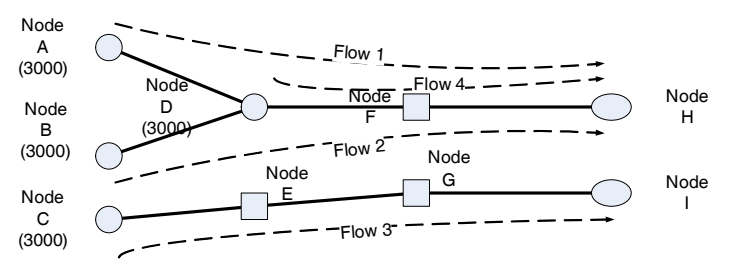

(c)

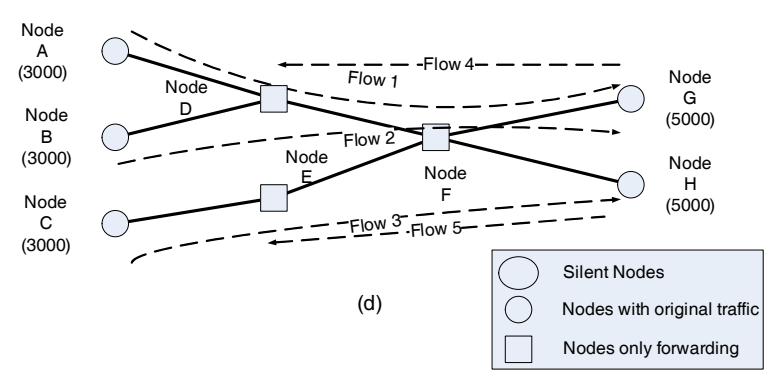

Fig. 4. The topologies for evaluation
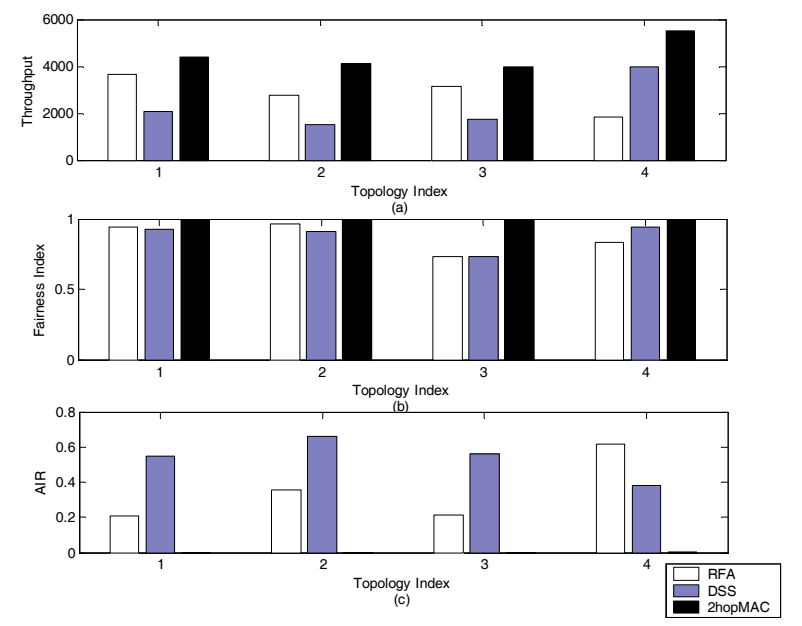

Fig. 5. Comparison of end-to-end throughput (a), fairness index (b) and AIR (c) for RFA, DSS and 2hopMAC schemes

The fairness index in the simulation is calculated in the following way:

$$
\zeta=\frac{\left(\sum X_{i}\right)^{2}}{n \cdot \sum\left(X_{i}\right)^{2}}
$$

where $X_{i}$ is the the ratio between the realized flow rate and the corresponding flow rate requested for flow $i$.

From Fig. 5, we can see the performance comparison for RFA, DSS and 2-hop MAC. In this figure, the benefits of end-to-end throughput and fairness of 2-hop MAC are shown 
by the comparison with RFA and DSS. Part (a) compares the end-to-end throughput performance. The proposed scheme has achieved significant gain in every topology setting. It is interesting to note that the end-to-end throughput of RFA is better than DSS in Topology (a), (b) and (c). The reason is that DSS does not consider the multi-hop property of the traffic, thus the spectrum allocation can deviate far from the ideal case. For Topology (d), because there is a center-like point, DSS can allocate the spectrum better than RFA. Note that the regulation mechanism for asymmetric neighborhood problem is assumed for RFA and DSS, and thus the realistic performance should be worse. The other comment for this simulation is that the traffic demand is intentionally set so that the total traffic demand barely exceeds the channel limit and each flow has identical demand. When the traffic demand of each flow and the difference of flow's traffic demand increase, the throughput performance gain of the proposed scheme can be higher because each node has more different traffic demand and a poor spectrum allocation can cause more significant throughput degradation.

Part (b) shows the fairness performance comparison. The fairness among different flows is guaranteed only with 2hop MAC. Since each node is assumed to deliver the traffic according to the proportion of the traffic from different sources, nodes which do not know the real traffic demand from different sources but the arrival traffic amount, cannot allocate the spectrum usage fairly according to their knowledge.

End-to-end throughput is not a good indicator of system's performance in the sense that it cannot tell how good a scheme is. It depends greatly on the traffic demand of each node as well as the flow distribution in the networks. AIR is introduced as the indicator of how good the spectrum is allocated using the given scheme. If AIR reaches 0 , the scheme allocates the system's spectrum to the serviced nodes without any wastage, which means the packets obtaining the spectrum to deliver at their first hop have obtained the virtually reserved spectrum for their following hops. There is no doubt that when the available spectrum is allocated without wastage, the efficiency of the spectrum allocation achieves its best performance. However, due to the complexity of topology and traffic distribution, non-zero AIR is usually inevitable. Part (c) shows the AIR comparison among 3 schemes, through which we can observe the spectrum allocation efficiency of each scheme. It can be seen that both RFA and DSS waste a lot of spectrum they allocate. Since DSS uses inaccurate traffic information to allocate spectrum, its AIR is higher than RFA's in some scenarios. For 2-hop MAC, AIR is almost 0 in all four topologies. This result is obvious in that each neighbor incorporates the exact traffic information from its neighbors and pass this information to the 2-hop neighbors via these neighbors. Through this method, the knowledge of accumulated traffic demand for each node in the neighborhood is correctly acquired. Note that in Topology (d), the AIR has a non-zero value because of the regulation for asymmetric neighborhood problem. This cannot be avoided when the distributed algorithm is applied since asymmetric neighborhood always exists in multi-hop wireless networks.

\section{CONCLUSION}

Throughput performance is always a key issue in multi-hop ad hoc networks. Distributed spectrum allocation/scheduling algorithms are commonly applied in the multi-hop networks to improve the efficiency of the spectrum usage and thus improve the poor throughput performance. However, without considering the multi-hop nature of flows, the spectrum allocation can have significant wastage especially for long hops flows. In this paper, we propose 2-hop MAC scheme. This scheme incorporates multi-hop consideration into spectrum allocation so that the spectrum allocated for one hop transmission will not be wasted due to lack of spectrum at the next hop. The asymmetric neighborhood, which is usually ignored in previous papers, is discussed and addressed in this paper. The endto-end throughput performance and fairness improvements are investigated in several typical multi-hop scenarios.

\section{REFERENCES}

[1] P. Gupta and P. Kumar, "The capacity of wireless networks," IEEE Trans. Inform. Theory, vol. 46, no. 2, pp. 388-404, Mar. 2000.

[2] H. Zhai, X. Chen, and Y. Fang, "Improving transport layer performance in multihop ad hoc networks by exploiting MAC layer information," IEEE Trans. Wireless Commun., vol. 6, no. 5, pp. 1692-1701, May 2007.

[3] H. Zhai, J. Wang, and Y. Fang, "Ducha: a dual-channel MAC protocol for mobile ad hoc networks," IEEE Trans. Wireless Commun., vol. 5, no. 11, Nov. 2006.

[4] S. Kim, B. Kim, and Y. Fang, "Downlink and uplink resource allocation in IEEE 802.11 wireless LANs," IEEE Trans. Veh. Technol., vol. 54, no. 1, pp. 320-327, Jan. 2005.

[5] H. Zhai and Y. Fang, "Distributed flow control and medium access control in mobile ad hoc networks," IEEE Trans. Mobile Computing, vol. 5, no. 11, pp. 1503-1514, Nov. 2006.

[6] X. Yang and N. Vaidya, "Priority scheduling in wireless ad hoc networks," Wireless Networks, vol. 12, no. 3, pp. 273-286, May 2006.

[7] V. Kanodia, C. Li, A. Sabharwal, B. Sadeghi, and E. Knightly, "Distributed priority scheduling and medium access in ad hoc networks," Wireless Networks, vol. 8, no. 5, pp. 455-466, Sept. 2002.

[8] X. Lin and S. Rasool, "A distributed joint channel-assignment, scheduling and routing algorithm for multi-channel ad hoc wireless networks," in Infocom'07, Anchorage, AK, May 2007.

[9] E. Modinao, D. Shah, and G. Zussman, "Maximizing throughput in wireless networks via gossiping," in SIGMetrics/Performance'06, Saint Malo, France, June 2006.

[10] V. Gambiroza, B. Sadeghi, and E. Knightly, "End-to-end performance and fairness in multihop wireless backhaul networks," in Mobicom'04, Philadelphia, PA, Oct. 2004.

[11] C. Joo and N. Shroff, "Performance of random access scheduling schemes in multi-hop wireless networks," in Infocom'07, Anchorage, AK, May 2007.

[12] Q. Dong, S. Banerjee, and B. Liu, "Throughput optimization and fair bandwidth allocation in multi-hop wireless LANs," in Infocom'06, Barcelona, Spain, Apr. 2006.

[13] B. Li, "End-to-end fair bandwidth allocation in multi-hop wireless ad hoc networks," in ICDCS'05, Columbia, OH, June 2005.

[14] T. Joshi, A. Mukherjee, Y. Yoo, and D. Agrawal, "Airtime fairness for IEEE 802.11 multirate networks," IEEE Trans. Mobile Computing, vol. 7, no. 4, pp. 513-527, Apr. 2008.

[15] H. Zhai, Y. Kwon, and Y. Fang, "Performance analysis of IEEE 802.11 MAC protocols in wireless LANs," Wireless Commun. Mobile Computing, vol. 4, no. 8, pp. 917-931, Nov. 2004. 